\title{
AMCP Partnership Forum: Improving Quality, Value, and Outcomes with Patient-Reported Outcomes
}

\section{SUMMARY}

Patient-reported outcomes (PROs), which provide a direct measure of a patient's health status or treatment preferences, represent a key component of the shift toward patient-centered health care. PROs can measure the state of a patient's disease-specific and overall health throughout the care continuum, enabling them to have a variety of uses for key health care stakeholders. Currently, PROs are used in drug development, aligning patient and clinician goals in care, quality-of-care measures, and coverage and reimbursement decisions. While there have been significant strides by key health care stakeholders to further the development and use of PROs, there are a number of challenges limiting more widespread use.

In light of these current challenges and the potential for PROs to improve health care quality and value, on October 19, 2017, the Academy of Managed Care Pharmacy convened a forum of key stakeholders representing patients, payers, providers, government, and pharmaceutical companies to discuss and identify solutions to the current challenges and barriers to further use of PR0s. These discussions informed the development of participants' ideal future state in which PR0s maximize the goals of all health care stakeholders and the actionable steps required to make the future state a reality. While stakeholders shared unique perspectives throughout the forum, they had consensus on 2 overarching issues: the importance of PROs in defining value, improving patient care, and implementing valuebased payment models and the need for strong organizational and operational systems to achieve optimal adoption and use.

Participants identified several key challenges in PRO use and adoption: achieving a representative patient population, inclusion of PRO data in medication labels, the necessity for both standardized and customizable PROs, and operational and organizational barriers to collecting and analyzing PROs. To overcome these challenges, participants recommended that manufacturers should engage key stakeholders early and throughout the drug development process to ensure the most valid and representative PROs and patient populations will be included. To streamline the PRO collection process, participants suggested engaging pharmacists and other providers who may have more frequent interaction with patients. Participants also recommended that PRO collection and analysis should use common technology platforms, streamline components of clinician care to reduce workflow, and be integrated with claims data to provider payers a better understanding of patient health in real time. Finally, additional work should be done to develop patient-reported outcome measures that contain relevant measures for all healthcare stakeholders. While significant challenges remain in PRO development and adoption, participants agreed that greater use can only be achieved through collaboration and patientcentered care.

J Manag Care Spec Pharm. 2018;24(3):304-10

Copyright $\odot 2018$, Academy of Managed Care Pharmacy. All rights reserved.
$\mathrm{P}$ atient-centered health care, which is responsive to an individual's preferences, needs, and values, is an essential component of new health care models that focus on quality and value..$^{1-3}$ Understanding patient needs and preferences can promote better patient care, subsequently improving decision making and resource utilization, driving broader policy and coverage decisions, and supporting research and development initiatives. This greater focus on patient-centricity has led to the development and use of patient-reported outcomes (PROs), which provide direct measures of patients' disease symptoms or characteristics that should be addressed and/ or maintained. ${ }^{4}$ To measure a patient's perception of health care quality, PROs can be used as patient-reported outcome measures (PROMs) and patient-reported outcome performance measures (PRO-PMs)., 5 Additionally, the ability to measure PROs can help health care stakeholders better allocate resources, define priorities, develop better treatment plans, inform the development of guidelines, and guide research to drive patient-centric care. The development of PROs has been promoted by initiatives such as the creation of the PatientCentered Outcomes Research Institute under the Patient Protection and Affordable Care Act. ${ }^{7}$

\section{Current Use of PROs}

PROMs can be leveraged to capture information on a patient's overall health and well-being, such as a holistic, single-item "patient global" score, and disease-specific symptoms and characteristics. Additionally, they can capture the benefits and risks (i.e., side effects) of specific treatments as perceived by patients. As a result, PROs have a wide variety of uses, including assessing symptom severity, informing treatment decisions, connecting clinicians to patient-generated data, tracking outcomes, prioritizing patient-clinician discussions, monitoring general health and well-being, and evaluating population health initiatives. ${ }^{8,9}$ In addition, they are used in workplace biometric health screenings to prevent and assess employees' health conditions. ${ }^{10}$ They are increasingly valued as endpoints in drug development to support labeling and registration.

In light of the current and growing use of PROs, several groups, including the U.S. Food and Drug Administration (FDA), have published guidance documents to help facilitate alignment on measure development, data validation and implementation, and reporting of PROs. ${ }^{11}$ In addition, several groups provide item banks, a notable example being the Patient-Reported Outcomes Measurement Information System (PROMIS), which provides PROMs for use in research and clinical settings for the general population and other populations with chronic conditions. ${ }^{12}$ 


\begin{tabular}{l|l}
\multicolumn{1}{c|}{ TABLE 1 } & Opportunities to Expand Uses of PROs \\
\hline Drug development & $\begin{array}{l}\text { Integration into clinical trial design and FDA treat- } \\
\text { ment label endpoints and product labels }\end{array}$ \\
\hline Clinical care & $\begin{array}{l}\text { - Integration into routine clinical assessment to align } \\
\text { clinician and patient treatment goals and track out- } \\
\text { comes between traditional visits } \\
\text { - Measurement to systematically capture and quan- } \\
\text { tify patient symptoms } \\
\text { - Tool to capture and promote clinician best practices }\end{array}$ \\
\hline $\begin{array}{l}\text { Formulary } \\
\text { and coverage } \\
\text { decision making }\end{array}$ & $\begin{array}{l}\text { Component of value-based models, such as value- } \\
\text { based contracting }\end{array}$ \\
\hline FDA=U.S. Food and Drug Administration; PRO=patient-reported outcome.
\end{tabular}

Although there have been significant strides to incorporate PROs as part of routine patient-centered care, challenges limit more widespread adoption and use. These include a clear and consistently defined methodology for developing, collecting, and using PROs, engaging patients throughout the entire PRO process, and limited technology infrastructure. Given the variety of current challenges, widespread engagement of health care stakeholders is needed to develop solutions and facilitate greater use of PROs.

\section{Goals of the Partnership Forum}

On October 19, 2017, the Academy of Managed Care Pharmacy (AMCP) convened a group of stakeholders representing patients, payers, providers, government, and pharmaceutical companies to discuss solutions for key challenges to the use of PROs. Specifically, the forum's aims were the following:

- Explore how PROs are used in drug development and quality performance standards.

- Address obstacles that affect PRO utilization.

- Identify an ideal future state where PROs are used to maximize the goals of all health care stakeholders.

- Determine what actionable steps need to be taken to make an ideal future state a reality.

Although the stakeholders shared unique perspectives, they found broad consensus on 2 critical issues. First, PROs are crucial in defining value, improving patient care, and implementing value-based payment models. Second, significant organizational and operational factors are needed to achieve the aforementioned adoption and use.

\section{Opportunities to Expand Current Use of PROs}

Forum participants discussed the current and potential uses of PROs within their organization (Table 1). Ideally, PRO collection and application should be aligned across health care stakeholders and settings of care delivery. There was widespread agreement that PROs should be integrated into clinical trial designs so that they may be part of FDA treatment label endpoints and subsequently drive care and coverage decisions. PROs also should capture patient health and well-being beyond clinical endpoints, including the ability to achieve social and personal goals, which often are more important to patients. PROs also can be better integrated into routine clinical care to better align clinician and patient priorities. For example, data from PRO collection can inform clinician best practices, which can be shared across the organization. Finally, stakeholders agreed that PROs could improve coverage decision making and be used as components of value-based models, such as valuebased contracting.

\section{Current Challenges in Implementing PROs}

\section{PROs in the FDA Medication Approval Process}

\section{and Quality Measures}

Participants recognized that PROs are an important component of precision medicine, or the customization of medical treatments to patient-level factors that influence treatment response. ${ }^{13}$ Some stakeholders expressed that verified and validated PROMs are as meaningful as other clinical measures for establishing outcomes. Further, patient preferences are now viewed as an important component in understanding and describing the value of a drug prior to the FDA approval process.

The Patient Population. Participants noted that the patient population involved during drug development stages must be representative of the broader population of interest. However, populations in clinical trials do not always fully represent patients who use the medications in real-world settings postapproval. While discussing the role of patients in developing and validating PROs used in drug development, participants noted that manufacturers should plan to include patients strategically within the initial stages of drug development, even prior to trial enrollment. Further consideration is necessary in this area and warrants additional work by forum participants and others. Given the sheer scale of wider PRO adoption across the disease spectrum, patients and their advocates will need to consider how to advance PRO use in conditions of greatest interest and need.

Prescription Drug Labeling. Several participants raised the importance of including PRO data in medication labels to support PRO use in clinical practice. PROs are often used as exploratory endpoints, which do not allow pharmaceutical companies to make claims about them, even if they provide valuable data. ${ }^{14}$ Participants also noted the challenges in achieving statistically significant and/or meaningful results of PRO endpoints in clinical trials and the need for PROs that are sensitive and directly related to a drug's clinical endpoints. As the FDA considers these issues, stakeholders from the forum should provide recommendations that will allow all participants to more effectively use PROs in clinical trials and medication labels. 
Data Collection. The current state of PROs in drug development and quality measurement requires intensive and ongoing data collection. Participants noted the challenge of information silos-which prevent best practices around PRO development, validation, and use-from being shared openly. Other participants discussed the need for greater collection of PRO data during drug development and clinical trials to better inform payer initiatives, like quality measurement. This challenge extends into clinical practice, where clinicians may be unsure of how to operationalize PRO data collection for routine use in clinical practice.

Recommendations. In light of these challenges, panel members noted the following ways that PROs can be better integrated across the spectrum of clinical trials, clinical practice, and quality measurement:

- To ensure sample populations are representative of the population indicated for a treatment, participants recommended an iterative process of validating PROs with broader networks of patients and using a mixed-methods approach to evaluate concordance across sample populations. ${ }^{15}$

- To facilitate the use of PROs early in the drug development process, manufacturers should engage patients and key stakeholders, such as the FDA and its Critical Path Innovation meetings. These meetings allow manufacturers to engage with the FDA during the drug development process to determine PROMs that are valid but also representative of patient needs.

- To better streamline PRO collection and ensure that useful data are being captured, participants suggested engaging pharmacists and other non-physician health care professionals, particularly as part of routine clinical care beyond the trial. Compared with clinicians who may be seen sporadically, other health care professionals who see patients more frequently (i.e., from picking up monthly medications) may have access to more information on patient factors like adherence and adverse events.

\section{Obstacles to Utilization of PROs in Patient Care}

When PROs are collected and analyzed in ways that are meaningful to patients, clinicians, and payers, they can enable better decision making, improve patient care, and quantify dimensions of care, such as daily living, that are traditionally difficult to measure and include in the care-planning process. Organizational and systemic barriers, however, prevent greater PRO utilization. Participants did, however, provide some ways to overcome these challenges.

Factors Related to PRO Collection and Analysis. Collecting and analyzing PROs requires significant effort, so organizations must commit to devoting time and resources to use PROs. Participants discussed their experiences engaging health care professionals in PRO collection and noted that this must be seamlessly integrated into current workflows, particularly if it can be done in a way to actually lessen staff time. Further, to keep clinicians engaged with PROs, data must be analyzed and shared quickly so that it can be actionable in patient care, facilitate clinicians' treatment decisions, and better inform coverage and reimbursement policies. Fundamentally, however, effective collection and analysis requires the development and validation of sensitive PROs.

Standardization Versus Customization. Participants discussed the necessity for PROs that are generalizable and customizable. The "good versus perfect" PRO was identified as a practical challenge for both patients and clinicians, as PRO questions may, to varying degrees, not align with every patient's personal experience. Participants noted the trade-off between wider use of a simple single-item PROM to inform the subsequent selection and use of condition-/treatment-specific PROMs. General PROMs, such as the EuroQol-5D (EQ-5D), ensure a level of standardization across stakeholders and health systems; however, they may not be sensitive to disease-specific characteristics or reflect the continuum of a patient's health state. ${ }^{16}$ For example, the EQ-5D measures 5 dimensions of health, mobility, self-care, usual activities, pain/discomfort, and anxiety/depression, but it cannot measure symptom burden and medication adherence for specific diseases like cancer or diabetes. ${ }^{8}$ Disease-specific PROs, conversely, can better capture the continuum of a patient's health state, but they often do not have the same validation.

Some participants also noted the need for PROs that are customizable to a patient's contextual factors or treatment setting to better achieve patient engagement with PRO collection. Ongoing work by AMCP and other stakeholders should explore the types of PROs that drive good patient outcomes and how to determine whether certain PROs should be standard or customized.

Predictive Versus Historical Perspective. Currently, PROs capture the present and/or past patient experience. This provides value on outcomes, such as treatment side effects, but often not a patient's future health state or resource use. Participants discussed the value of PROs with predictive ability, which could inform patients and clinicians about future health states and resource use, and subsequently improve patient outcomes by providing opportunities for interventions. Work by AMCP and other key stakeholders also should provide recommendations on developing and evaluating predictive PROs.

Technology Barriers. Current methods for PRO collection can be time and resource intensive for both patients and clinicians. As a result, there is a need for PRO collection methods that do not affect clinicians' workflow and are accessible and easy to use for patients. This has clear implications on providers of electronic health record (EHR) technologies. Discussions around technological barriers led some participants to discuss 
the broader challenge of maximizing the application of technology to passively capture social and demographic characteristics of patients.

Recommendations. Panel members recommended the following:

- PRO collection may augment existing components of a clinician's assessment to reduce workflow burden and improve patient outcomes. For example, PROs could be better integrated into EHRs to provide specific actions for clinicians, such as flagging the need to change a patient's treatment because of the occurrence of an adverse event; however, there is limited ability to do so in the current state.

- To ensure PRO data are actionable for clinicians, clinical sites should ensure that there are supporting operational and organizational resources.

- Through EHRs, PROMs should be better integrated with claims data to provide payers with a more comprehensive view of patients' health and treatment in real time and support formulary and coverage determinations. Such integration can allow payers to better track patient response and patterns of treatment.

- Use accessible and common technology platforms to collect PROs, such as smartphones, to minimize the burden on patients and prevent the need for PROM-specific technologies. Allocate resources for hard-to-reach patients to promote engagement across all populations.

- To better inform treatment decisions and optimize health outcomes, PROMs should be developed with a greater emphasis on predictive ability, in addition to the past or current health state.

\section{The Future State of PROs}

\section{Creating the Future State}

Participants assumed the roles of key PRO stakeholders (i.e., patients/advocacy organizations, clinicians, payers, and manufacturers) to identify priorities for a future ideal state of PRO utilization.

Patient/Advocacy Perspective. From a patient perspective, participants noted that PROMs should be simple, customizable, and representative of real outcomes. Further, PROMs should take into account patient literacy and contextual factors. The PROM collection process for patients should be manageable to avoid survey fatigue and promote engagement. For example, PROs can be collected on patients as part of the routine intake process at a provider's office. PRO data should be returned to patients in an understandable format and reflect the entire disease or health state so that patients can be better educated about their own health status and make more informed healthrelated decisions.

Clinician Perspective. Many of the priorities from the clinician perspective focused on standardized collection and technology. Strong information technology (IT) and data

\section{TABLE 2 Priorities of Key PRO Stakeholders}

\begin{tabular}{|c|c|}
\hline Stakeholder Group & Priorities for the Ideal State \\
\hline Patients/advocacy & $\begin{array}{l}\text { - Simple, customizable PROs that reflect patient lit- } \\
\text { eracy and contextual factors } \\
\text { - PRO data that are presented in an understandable } \\
\text { way to promote informed treatment goals and } \\
\text { decision making }\end{array}$ \\
\hline Clinicians & $\begin{array}{l}\text { - Standardized data collection and technology } \\
\text { - PROs that can facilitate care decisions, streamline } \\
\text { care, and align patient and clinician goals }\end{array}$ \\
\hline Payers & $\begin{array}{l}\text { - Integration of PROs into initiatives, such as value- } \\
\text { based contracting, to support the appropriate use } \\
\text { of treatments } \\
\text { - PROs that can generate information relevant to all } \\
\text { stakeholders to better inform and support coverage } \\
\text { decisions throughout a drug's life cycle }\end{array}$ \\
\hline Manufacturers & $\begin{array}{l}\text { - Widespread use in clinical trials to support the } \\
\text { drug approval process } \\
\text { - PROs that are representative of the patient popula- } \\
\text { tion and usable in real-world settings }\end{array}$ \\
\hline
\end{tabular}

$P R O=$ patient-reported outcome.

collection systems are required to facilitate data sharing among stakeholders. To ensure participation in PRO collection, some participants suggested that clinicians should be given financial incentives or similar benefits. Across all participants, it was agreed that PROs should be a tool to facilitate care decisions and align patient and clinician treatment goals.

Payer Perspective. The ideal state of PROs for payers focused around providing value and improving patient health. The payer perspective also prioritized PROs that were universally useful for researchers, clinicians, and payers, as few types (e.g., health-related quality of life vs. proximal effects) of PROs are relevant to all stakeholders. Some participants provided specific examples by which PROs could inform decision making and resource utilization, such as the cost-effectiveness of a therapy and decisions around access to a treatment. There was consensus that PRO data should be incorporated into clinical care plans and pathways. A theme in this session, and throughout the day, was the potential role of PROs in valuebased contracting to demonstrate the efficacy and appropriate indication of a therapy.

Manufacturer Perspective. All groups noted that PROMs should be integrated into clinical trial designs and that the ideal state would be more widespread use of PROM endpoints to support drug approval to enable meaningful comparisons across drugs. Further, there must be consensus among functional units within a manufacturer on the role and importance of PROMs. Some participants commented that PROMs used by manufacturers must be scalable and representative of the broad patient population and also usable in a real-world 


\section{Current Challenges}

\section{Ideal State of PROs}

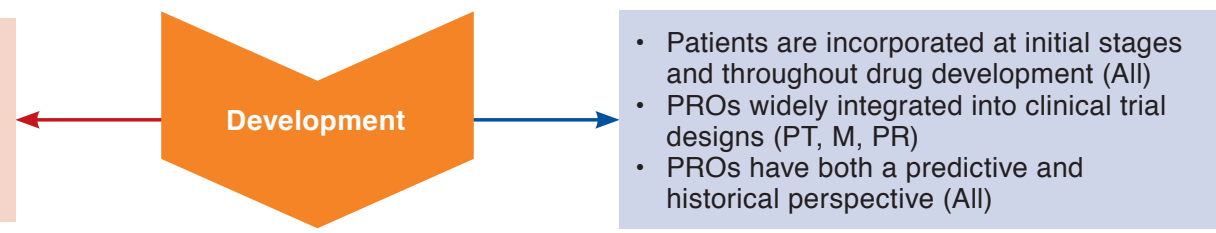

Lack or representative patient population involved during drug development (M, PT)

- PROs largely capture current or historical health states, limiting their ability to predict future states (All)

- Information silos are preventing sharing of best methodological practices (M, C, PR)

- Tension between validated general PROs and those that can be customized to disease or social characteristics (All)

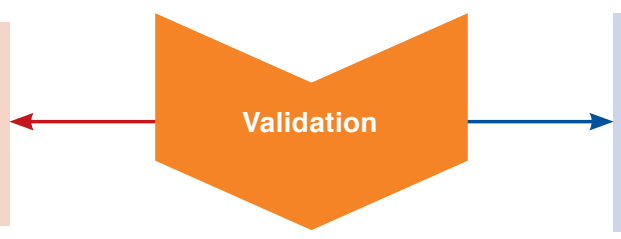

- Common terminology for PRO best practices (All)

PROs that are simple and validated yet customizable (All)

- PROs that are clinically sensitive and medically relevant (All)

Lack of broad integration into clinical and coverage decision making (M, C, PR)

- Lack of buy-in within and across organizations (M, C, PR)

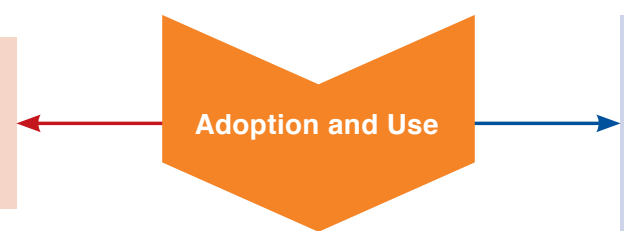

- PRO collection that is streamlined and efficient throughout a patient's health or disease state (All)

- Widespread integration of PRO endpoints into manufacturer labels (PT, M, PR)

Data not shared across stakeholders to inform decision making (All)

- Current methods for collection and analysis can be time-consuming for stakeholders (M, C, PR)

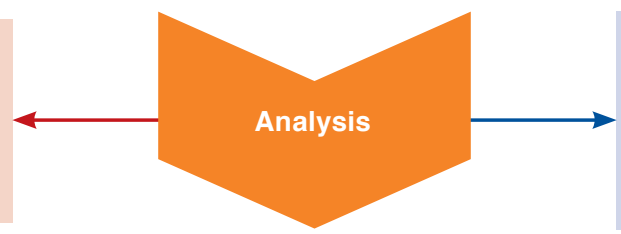

A single platform for PRO collection and analysis (M, C, PR)

Clinician and payer incentives align with PRO measurement and analysis (C, PR)

PROs inform treatment decisions (All)

All = all stakeholders; $C=$ clinician; $M=$ manufacturer $; P R=$ payer $; P T=$ patient/advocacy.

setting. Finally, participants noted that data between clinicians and manufacturers should be shared to help drive decision making and for understanding the effect of treatments in realworld settings.

Ideal State of PROs Across All Stakeholders. Although each stakeholder group had unique priorities (Table 2), some priorities were overarching and spanned the groups (Figure 1):

- There must be common terminology to facilitate an understandable and meaningful dialogue between stakeholders, which may include best practices for PRO development, collection, analysis, and reporting

- Collection and utilization should be streamlined and efficient so as not to add an additional burden on stakeholders.

- PROs should be both standardized and customized to enable comparisons while reflecting specific disease states and patient needs.

- PROs should be better integrated into drug approval labels to facilitate meaningful use by stakeholders, including patients, manufacturers, payers, and clinicians

\section{Making the Future State a Reality}

A Common Language. Participants agreed that a common framework of PRO development, collection, analysis, reporting, and utilization is needed as a first action to promote the more widespread diffusion of education, knowledge, and expertise. Although organizations, such as the National Quality Forum, have developed definitions of PROs and associated terms, there must be consensus. ${ }^{5}$ Otherwise, there is a lack of agreement among stakeholders about what to measure, analyze, and report. This framework also should include best practices in applying PRO data to care delivery decisions and coverage determinations. Participants also noted that sharing the methodology used for developing and analyzing PROs is valuable to all stakeholders and should be openly shared.

Meaningful Measures. PROs should reflect how patients perceive their own health or disease state while also representing what clinicians view as clinically relevant. To achieve representativeness of both patients and clinicians, PROs should be tested with small groups and repeatedly validated with broader networks. Further, PROs should be able to demonstrate a return on investment to promote stakeholder use. This could be 
achieved through dissemination of research or engaging other stakeholders. All participants agreed that meaningful PROMs are those that are actionable and can provide real-time insight on interventions or treatments to improve patient health.

Technology and Infrastructure. Finally, participants discussed the need for infrastructure and IT systems to better support the collection and analysis of PROMs. This includes integration of general and disease-specific PROMs into EHRs to better facilitate care delivery and decision making. Such integration would provide clinicians and payers with a broader view of a patient's health by integrating clinical outcomes with those reported to be important by the patient. Some participants also suggested a single platform or system be used by all stakeholders to enable standardization of PROM development, collection, analysis, and reporting.

\section{Conclusions}

Among all key health care stakeholders, PROs are considered important in fostering patient-centric care in drug development, formulary decision making, and treatment care pathways in clinical practice. While there has been greater attention to integrating PROs in drug development, clinical care, and coverage determination and reimbursement, there are considerable barriers for all stakeholders that are limiting greater use. In light of these barriers, forum participants developed a number of key recommendations. Fundamentally, health care stakeholders must collaborate on best practices for developing simple and meaningful PROs that can support drug development, align patient/clinician decision making, and better inform coverage and reimbursement decisions. Across all stakeholders, PRO use will only be successful, however, if they represent meaningful patient-driven measures of care.

\section{Forum Participants}

AMANDA BAIN, PharmD, MPH, MBA, Director, Pharmacy and Care Management, The Ohio State University Health Plan; KRISTEN BINASO, RPh, Director, Patient Advocacy and Professional Relations, Boehringer Ingelheim Pharmaceuticals; ROBYN CARSON, MPH, Executive Director, Patient-Centered Outcomes Research, Allergan; DEB CURRY, PharmD, Senior Director, Formulary Product Strategy, OptumRx; BAHAR DAVIDOFF, PharmD, Vice President, Pharmacy, Regal Medical Group; MICHELE V. DAVIDSON, RPh, Senior Manager, Pharmacy Technical Standards, Policy \& Development, Walgreens; JESSICA DAW, PharmD, MBA, Senior Director, Clinical Pharmacy, UPMC Health Plan; JOSEPH DICESARE, Health Economics and Outcomes Research Head, Neuroscience, Respiratory, E Ophthamology, Novartis Pharmaceuticals; SARAH DONELSON, MA, Lead, Patient-Centered Research \& Collaboration, Genentech; SETH GINSBERG, Co-founder, Global Healthy Living Foundation; DOUGLAS GOLDSTEIN, AVP, Innovation Officer, Inova Center for Personalized Health; HILARY HATCH, PhD, Founder and Chief Executive Officer, Vital Score; JOHN KALADA, Vice President, Market Access Consulting and Communications, Xcenda; FRANCIS KALUSH, PhD, Programs Health Coordinator, U.S. Food and Drug Administration, Center for Drug Evaluation and Research; ROSS MACLEAN, MD, SVP, Head of Medical, Precision Health Economics; ROBERT MCBURNEY, PhD, Chief Executive Officer, Accelerated Cure Project for Multiple Sclerosis; MICHELLE MOCARSKI, MPH, Director, Health Economics and Outcomes Research, Novo Nordisk; DAVID NILASENA, MD, MSPH, MS, Chief Medical Officer, Region VI, Centers for Medicare \& Medicaid Services; LISA OPIPARI-ARRIGAN, PhD, Associate Professor, Cincinnati Children's Hospital Medical Center; MATTHEW PICKERING, PharmD, Associate Director, Research \& Quality Strategies, Pharmacy Quality Alliance; AISHA PITTMAN, MPH, Senior Director, Quality Policy, Premier; LOU SANQUINI, Vice President, Healthagen; SANDHYA SAPRA, PhD, Director, Amgen; RACHEL CLARK SISODIA, MD, Assistant Professor and Medical Director, Massachusetts General Physician Organization, Harvard Medical School/Massachusetts General Hospital; JEAN SLUTSKY, MSPH, Chief Engagement and Dissemination Officer, Patient-Centered Outcomes Research Institute; TRACY SPINKS, BBA, Senior Director, Quality Innovation, National Quality Forum; RICHARD STANFORD, PharmD, MS, Senior Director, U.S. Value Evidence and Outcomes, GlaxoSmithKline; SHEILA THOMAS, PharmD, RPh, Senior Director, Global Health Economics and Value Assessment, Sanofi; ROBIN TURPIN, PhD, Value Evidence Lead, Takeda; MITZI WASIK, PharmD, BCPS, Stars Strategy Lead, Aetna; and LAURIE WESOLOWICZ, PharmD, FAMCP, Vice President, Payor Strategies, Diplomat Specialty Pharmacy.

CORRESPONDENCE: Caroline Huber, MPH, Precision Health Economics, 11100 Santa Monica Blvd., Ste. 500, Los Angeles, CA 90025. Tel.: 310.984.7717;

E-mail: Caroline.Huber@precisionhealtheconomics.com. 


\section{DISCLOSURES}

This AMCP Partnership Forum titled "Improving Quality, Value, and Outcomes with Patient-Reported Outcomes" and the development of this proceedings report were supported by Amgen, Boehringer Ingelheim Pharmaceuticals, Genentech, GlaxoSmithKline, Novartis Pharmaceuticals, Novo Nordisk, Precision for Value, Premier, Sanofi, Takeda Pharmaceuticals USA, and Xcenda.

\section{ACKNOWLEDGMENTS}

This proceedings report was written by Caroline Huber, MPH, of Precision Health Economics, which receives financial support for its research studies from pharmaceutical and other life sciences companies.

\section{REFERENCES}

1. Epstein RM, Street RL. The values and value of patient-centered care. Ann Fam Med. 2011;9(2):100-03.

2. Dardess P. Principles for making health care measurement patient-centered. 2017. Available at: https://aircpce.org/projects/developing-principlespatient-centered-measurement. Accessed January 29, 2018.

3. Baker A. Crossing the quality chasm: a new health system for the $21 \mathrm{st}$ century. Br Med J. 2001;323(7322):1192.

4. U.S. Food and Drug Administration. Guidance for industry: patientreported outcome measures: use in medical product development to support labeling claims. Fed Regist. 2009;74(235):65132-33.

5. Deutsch A, Smith L, Gage B, Kelleher C, Garfinkel D. Patient-reported outcomes in performance measurement. Paper presented at: National Quality Forum (NQF); October 22, 2012; Washington, DC.

6. Weldring T, Smith SM. Patient-reported outcomes (PROs) and patientreported outcome measures (PROMs). Health Serv Insights. 2013;6:61.
7. House Office of the Legislative Counsel. Compilation of Patient Protection and Affordable Care Act: extracted sections concerning Patient-Centered Outcomes Research and the authorization of the Patient-Centered Outcomes Research Institute (PCORI). 2010. Available at: https://www.pcori.org/sites/ default/files/PCORI_Authorizing_Legislation.pdf. Accessed February 5, 2018.

8. Lavallee DC, Chenok KE, Love RM, et al. Incorporating patient-reported outcomes into health care to engage patients and enhance care. Health Aff. 2016;35(4):575-82.

9. Hendrikx RJ, Spreeuwenberg MD, Drewes HW, Ruwaard D, Baan CA. How to measure population health: an exploration toward an integration of valid and reliable instruments. Population Health Manag. December 6, 2017 [Epub ahead of print]. Available at: http://online.liebertpub.com/doi/ abs/10.1089/pop.2017.0097?url_ver=Z39.88-2003\&rfr_id=ori\%3Arid\% 3Acrossref.org\&rfr_dat=cr_pub\%3Dpubmed\&. Accessed February 5, 2018.

10. Arena R, Arnett DK, Terry PE, et al. The role of worksite health screening. Circulation. 2014;130(8):719-34.

11. Basch E, Spertus J, Dudley RA, et al. Methods for developing patientreported outcome-based performance measures (PRO-PMs). Value Health. 2015;18(4):493-504.

12. National Institutes of Health, Office of Strategic Coordination-The Common Fund. Patient-Reported Outcomes Measurement Information System (PROMIS) Program Snapshot. Available at: https://commonfund.nih. gov/promis/index. Accessed January 29, 2018.

13. U.S. Food and Drug Administration. Paving the Way for Personalized Medicine: FDA's Role in a New Era of Medical Product Development. Silver Spring, MD: U.S. Food and Drug Administration; 2013.

14. Basch E. Toward patient-centered drug development in oncology. New Engl J Med. 2013;369(5):397-400.

15. Magasi S, Ryan G, Revicki D, et al. Content validity of patient-reported outcome measures: perspectives from a PROMIS meeting. Qual Life Res. 2012;21(5):739-46.

16. Brazier J, Roberts J, Tsuchiya A, Busschbach J. A comparison of the EQ-5D and SF-6D across seven patient groups. Health Econ. 2004;13(9):873-84. 